\title{
The comparison of the effectiveness between different doses of local methylprednisolone injection versus triamcinolone in Carpal Tunnel Syndrome: a double-blind clinical trial
}

This article was published in the following Dove Medical Press journal:

Journal of Pain Research

\section{Afshin Karimzadeh' \\ Shahriar Bagheri ${ }^{2}$ \\ Seyed Ahmad Raeissadat ${ }^{3}$ \\ Shahab Bagheri ${ }^{4}$ \\ Seyed Mansoor Rayegani ${ }^{4}$ \\ Shahram Rahimi-Dehgolan ${ }^{5}$ \\ Farshad Safdari ${ }^{2}$ \\ Hashem Abrishamkarzadeh ${ }^{2}$ \\ Hadi Shirzad ${ }^{6}$ \\ 'Department of Physical Medicine and Rehabilitation, Clinical Research \\ Development Center of Imam-Hossein Educational Hospital, School of Medicine, Shahid Beheshti University of Medical Sciences, Tehran, Iran; ${ }^{2}$ Department of Orthopedic Surgery, Bone, Joint and Related Tissue Research Center, Akhtar Educational Hospital, Shahid Beheshti University of Medical Sciences, Tehran, Iran; ${ }^{3}$ Clinical Development Research Center of Shahid Modarres Hospital, Physical Medicine and Rehabilitation Department and Research Center, School of Medicine, Shahid Beheshti University of Medical Sciences, Tehran, Iran; ${ }^{4}$ Physical Medicine and Rehabilitation Department and Research Center, Shohada-e-Tajrish Hospital, School of Medicine, Shahid Beheshti University of Medical Sciences, Tehran, Iran; ${ }^{5}$ Department of Physical Medicine and Rehabilitation, School of Medicine, Tehran University of Medical Sciences, Tehran, Iran; ${ }^{6}$ Department of Medical Genetics, Tarbiat Modares University, Tehran, Iran}

Correspondence: Seyed Ahmad Raeissadat Clinical Development Research Center of Shahid Modarres Hospital, Physical Medicine and Rehabilitation Department and Research Center, School of Medicine, Shahid Beheshti University of Medical Sciences, No. 1998734383, Shahid Modarres Hospital, Kaj Square, Saadat Abad Street, Tehran, Iran Tel +98212 2074090 Fax +98 2122074090 Email a.raeissadat@gmail.com

\begin{abstract}
Purpose: Local corticosteroid injection is one of the most prevalent methods in treating carpal tunnel syndrome (CTS). However, the most efficient substance and its appropriate dosage remain controversial. In the present double-blind randomized controlled trial, the efficacy and safety of local injection of two corticosteroids (triamcinolone and methylprednisolone) were compared at two different dosages, 20 and $40 \mathrm{mg}$.
\end{abstract}

Patients and methods: We consecutively included 80 patients with mild or moderate CTS and randomly assigned them to four groups: 20 or $40 \mathrm{mg}$ triamcinolone (T20 or T40) and 20 or $40 \mathrm{mg}$ methylprednisolone (M20 or M40) groups; each patient received a single injection of steroid using conventional approach. The four groups were relatively comparable and did not show any significant difference initially in their baseline measurements including pain intensity measured using VAS, pain-free grip strength (PFGS), nerve conduction study (NCS), and two parts of Boston Carpal Tunnel Syndrome Questionnaire: symptom severity scale (SSS) and functional status scale (FSS); the latter was our primary outcome measure. Three months after injection, they were reassessed to evaluate the clinical and electrodiagnostic changes.

Results: Almost all NCS parameters, VAS, and PFGS significantly improved after treatment in all the groups $(P<0.05)$. Compound motor action potential amplitude significantly improved only in T40 group ( $P=0.032$ ), while there was no significant improvement in other groups. Furthermore, SSS remarkably decreased in all the four groups, without any significant difference between the groups $(P=0.87)$. A similar significant decrease was found in FSS, with a higher improvement in T40 group $(P=0.009)$. There was no significant difference between the four groups in other variables after treatment.

Conclusion: Based on the current data, the efficacy and safety of local injection of triamcinolone and methylprednisolone at doses of 20 and $40 \mathrm{mg}$ were associated with a significant improvement in pain, functional status, and strength. Although, there was no remarkable superiority, $40 \mathrm{mg}$ injection, especially for triamcinolone, yielded better NCS results and functional status Keywords: steroids, injections, conservative treatment, wrist

\section{Introduction}

Carpal tunnel syndrome (CTS) or compressive neuropathy of the median nerve under the flexor retinaculum of the wrist is the most common entrapment neuropathy. ${ }^{1}$ The incidence of CTS has been reported to be $6.7 \%-7.8 \% .^{2-4}$ Currently, there is no consensus about the appropriate treatment of CTS at different stages of the condition. ${ }^{5}$ In general, the surgical release of the flexor retinaculum is recommended to be done when the disease is advanced with severe symptoms or atrophy in physical examina- 
tion. ${ }^{6}$ Nonoperative treatments should be considered in mild to moderate cases. ${ }^{7-12}$

Previously, only few studies have compared the efficacy of local injection of different substances in patients with CTS. ${ }^{13-18}$ Corticosteroids, especially methylprednisolone and triamcinolone, are among the most prevalent medications used for local injection in patients with CTS. ${ }^{13-15}$ The therapeutic mechanism of local corticosteroid injection (CSI) has been speculated via a probable suppression of inflammation and a subsequent decompression of the median nerve. CSI is well established and the most rapid injection with a documented short-term efficacy, but there is inadequate evidence over the best choice of steroid and dose..$^{819-21}$ Evidently, the complications of local corticosteroids should be cautiously considered, in particular regarding diabetic patients. ${ }^{20}$

Regarding the extensive variety of treatments, as well as their combinations, and due to the lack of comprehensive meta-analyses in view of different study designs and various patient characteristics, the most efficient steroid and its most appropriate dosage that would be associated with best outcomes and less complications remain controversial. In the current randomized controlled trial (RCT), the efficacy and safety of local injection of methylprednisolone and triamcinolone at two different doses of 20 and $40 \mathrm{mg}$ were compared within a follow-up period of 3 months, in patients with mild to moderate CTS.

\section{Patients and methods}

During the first 6 months of 2017 from January to June, 112 patients referred to the Electrodiagnosis Unit of Shohada-eTajrish Hospital with complaints related to one of the main symptoms of CTS were consecutively recruited. Sampling continued until 80 electrodiagnostically confirmed CTS cases of mild or moderate stages were included. A written informed consent was obtained from all the eligible patients in compliance with the Declaration of Helsinki. Then, they were assigned to four groups of 20 members each using block randomization method (block size $=4$ members): 20 $\mathrm{mg}$ triamcinolone (T20), $40 \mathrm{mg}$ triamcinolone (T40), $20 \mathrm{mg}$ methylprednisolone (M20), and $40 \mathrm{mg}$ methylprednisolone (M40).

All the included patients were symptomatic, as they had at least one of the major symptoms of CTS (pain, numbness, paresthesia in the palm or fingers, and subjective weakness in grip). The symptoms should have been lasting for 3-12 months and not been relieved by other conservative treatments such as night splinting and physical agent modalities such as laser therapy, ultrasonography (US), and manual therapy. ${ }^{22}$ The exclusion criteria were severe stage of CTS; current pregnancy; any history of diabetes mellitus, rheumatoid arthritis, or thyroid disorders; previous history of relevant surgery or hand trauma; any local injection in wrist within the last 6 months; thenar atrophy in physical examination; and active cervical radiculopathy or other peripheral neuropathies in the upper extremity.

In the case of bilateral involvement, only the side with more severity was included.

Furthermore, if electrodiagnostic studies did not confirm the presence of CTS, the patient was excluded. The patients and also the physicians who were responsible for injection and evaluation (electrodiagnostic and clinical measurements) were all blinded to the groups. The syringes were covered by aluminum envelopes to hide the materials. The Institutional Review Board and Ethics Committee of Shahid Beheshti University of Medical Sciences approved the study protocol (Number: IR.SBMU.MSP.REC.1396.157). We also registered this trial in the Iranian Registry of Clinical Trials (ID number: IRCT20130523013442N22).

The demographic characteristics of the patients such as age, gender, height, weight, body mass index (BMI), and the severity grade of disease were recorded. The clinical outcome-measuring tools were as follows: 1) a 10-score VAS in which 0 indicated "no pain" while 10 indicated the "maximal imaginable pain", which was used to assess the pain intensity; 2) Boston Carpal Tunnel Syndrome Questionnaire (BCTQ), which had two sections: symptom severity scale (SSS) of eleven items and functional status scale (FSS) of eight items. Each of these 19 questions had five choices based on the severity of involvement; higher scores indicated more severity of the condition; 3) pain-free grip strength (PFGS) utilizing dynamometer per kilogram; and 4) electrodiagnostic studies of median nerve measuring the amplitude (= amp) and latency of compound motor action potential (CMAP), latency of sensory nerve action potential (SNAP), and nerve conduction velocity (NCV) for motor fibers. In the electrodiagnostic evaluation, the criteria for the diagnosis of "mild" CTS were SNAP latency $>3.6 \mathrm{~ms}$ with preserved SNAP amp, normal CMAP amp, and latency, while criteria for the diagnosis of "moderate" CTS were SNAP latency $>3.6$ $\mathrm{ms}$ in addition to CMAP latency of between 4.2 and $6.5 \mathrm{~ms}$, with a preservation of SNAP. ${ }^{23}$ The first two variables (VAS and BCTQ) were our primary outcome-measuring tools, but PFGS and electrodiagnostic parameters were also evaluated as the secondary outcomes.

The local steroid injection was performed using the assigned substance for each group by the same physiatrist 
with a 20 years of expertise in the field of neuromuscular injections (the first author), under sterile condition, via landmark-guided method and without the guidance of US. The patients were lying supine while the wrist was placed on a table in resting position with slight wrist dorsiflexion. A 25-G needle was inserted in the volar surface of the wrist, above the distal wrist crease, between the two tendons of palmaris longus and flexor carpi radialis. After skin preparation, based on the conventional midline approach for CTS, injection was performed by passing the needle through the flexor retinaculum into the carpal tunnel. If the aspiration was negative, $1 \mathrm{~mL}$ of the drug was administered in combination with $1 \mathrm{~mL}$ of $2 \%$ lidocaine. After injection, all the patients remained in the clinic for at least half an hour to observe any severe reactions. They were advised to apply some lifestyle modifications (avoiding repetitive fine hand movements, lifting heavy objects, or prolonged flexed wrist position) and use acetaminophen per need, only within the next 48 hours. A senior physical medicine resident (the fourth author, SB) was responsible for having a phone call with the patients 3 and 10 days after injection. The patients were also asked to attend clinical and electrodiagnostic reassessment in the clinic after 3 months, in which the preinjection measurements (VAS for pain, PFGS, two parts of BCTQ, and electrodiagnostic parameters) were taken repeatedly. Data analysis was performed using SPSS statistical software Version 22.0. One-way ANOVA and Tukey's honest significant difference test were used to compare the quantitative variables between the four groups. To compare the pre- and posttreatment variables in each group, Wilcoxon test or paired $t$-test was used. The significance level was considered at $P<0.05$.

\section{Results}

Among the 80 eligible participants, 7 patients did not return to the last visit due to personal problems or other reasons, none of which were related to our injections or complications. Therefore, the study was completed with 73 patients: 18 ,
20, 17, and 18 patients in T20, T40, M20, and M40 groups, respectively. Initially, the four groups were relatively similar in terms of age, gender, BMI, and the severity grade of the condition (Table 1). As can be observed in Table 2, between the two parts of BCTQ (SSS and FSS), SSS significantly decreased in all the groups after the injection, without any significant difference between them. The other score (FSS) also significantly decreased in the four groups; however, the improvement was significantly higher in $\mathrm{T} 40$ group compared with T20 group (Tukey`s $P=0.009$ ).

Table 2 Comparison of pre- and posttreatment clinical values in each group and between groups

\begin{tabular}{|c|c|c|c|}
\hline & Pre & Post & $\begin{array}{l}P \text {-value } \\
\text { (paired } t \text {-test) }\end{array}$ \\
\hline \multicolumn{4}{|l|}{ PFGS (kg) } \\
\hline$T 20(n=18)$ & $25.1 \pm 8.5$ & $30.6 \pm 10.9$ & $0.007^{*}$ \\
\hline $\mathrm{T} 40(\mathrm{n}=20)$ & $28.9 \pm 12.8$ & $33.1 \pm 14.4$ & $0.00 I^{*}$ \\
\hline$M 20(n=17)$ & $29.3 \pm 10.5$ & $35.7 \pm 19.8$ & 0.061 \\
\hline$M 40(n=18)$ & $24.6 \pm 10.7$ & $29.4 \pm 11.9$ & $0.00 I^{*}$ \\
\hline$P$-value (ANOVA) & 0.421 & 0.369 & \\
\hline \multicolumn{4}{|c|}{ VAS for pain (0-10 scale) } \\
\hline$T 20(n=18)$ & $5.4 \pm 2.4$ & $4 \pm 1.8$ & $0.007^{*}$ \\
\hline $\mathrm{T} 40(\mathrm{n}=20)$ & $5.4 \pm 2.4$ & $3.7 \pm 2.2$ & $0.001 *$ \\
\hline$M 20(n=17)$ & $5.6 \pm 1.8$ & $4.1 \pm 1.9$ & $0.014 *$ \\
\hline$M 40(n=18)$ & $5.3 \pm 2.9$ & $3 \pm 2.2$ & $0.006 *$ \\
\hline$P($ ANOVA $)$ & 0.975 & 0.474 & \\
\hline \multicolumn{4}{|l|}{ SSS (I I-55 scale) } \\
\hline$T 20(n=18)$ & $32.5 \pm I I .8$ & $24.9 \pm 10.2$ & $0.002 *$ \\
\hline $\mathrm{T} 40(\mathrm{n}=20)$ & $31.7 \pm 8.6$ & $22.3 \pm 11.1$ & $0.00 I^{*}$ \\
\hline$M 20(n=I 7)$ & $31.9 \pm 8$ & $22.8 \pm 8.5$ & $0.00 I^{*}$ \\
\hline$M 40(n=18)$ & $32.2 \pm 9.3$ & $22.6 \pm 7$ & $0.00 I^{*}$ \\
\hline$P($ ANOVA $)$ & 0.995 & 0.878 & \\
\hline \multicolumn{4}{|l|}{ FSS ( $8-40$ scale) } \\
\hline$T 20(n=18)$ & $22.6 \pm 9.9$ & $17.1 \pm 6.9$ & $0.00 I^{*}$ \\
\hline $\mathrm{T} 40(\mathrm{n}=20)$ & $21.8 \pm 7.3$ & $11.3 \pm 2.8$ & $0.001 *$ \\
\hline$M 20(n=17)$ & $19.2 \pm 6.5$ & $15.8 \pm 4.5$ & $0.001 *$ \\
\hline$M 40(n=18)$ & $23.2 \pm 9.6$ & $15 \pm 5.9$ & $0.002 *$ \\
\hline$P($ ANOVA $)$ & 0.528 & $0.01 I^{*}$ & \\
\hline
\end{tabular}

Note: *indicates significant $P$-value.

Abbreviations: FSS, functional status scale; M20, 20 mg methylprednisolone; M40, 40 mg methylprednisolone; PFGS, pain-free grip strength; T20, 20 mg triamcinolone; T40, $40 \mathrm{mg}$ triamcinolone; SSS, symptom severity scale.

Table I Comparison of demographic characteristics and baseline variables among the patients of the four groups

\begin{tabular}{|c|c|c|c|c|c|c|}
\hline & & T20 $(n=18)$ & T40 $(n=20)$ & $M 20(n=17)$ & $M 40(n=18)$ & $P$-value \\
\hline \multicolumn{2}{|l|}{ Age (years) } & $56 \pm 8.5$ & $54.8 \pm 7.6$ & $52.3 \pm 8.8$ & $57.8 \pm 6.6$ & 0.236 \\
\hline \multicolumn{2}{|l|}{ BMI $\left(\mathrm{kg} / \mathrm{m}^{2}\right)$} & $28.14 \pm 3.4$ & $26.4 \pm 2.7$ & $25.6 \pm 2$ & $27.7 \pm 4$ & 0.132 \\
\hline \multirow[t]{2}{*}{ Gender } & Male & 5 & 6 & 5 & 7 & \multirow[t]{2}{*}{0.291} \\
\hline & Female & 13 & 14 & 12 & 11 & \\
\hline \multirow[t]{2}{*}{ CTS severity } & Mild & 6 & 7 & 5 & 7 & \multirow[t]{2}{*}{0.197} \\
\hline & Moderate & 12 & 13 & 12 & 11 & \\
\hline
\end{tabular}

Abbreviations: BMI, body mass index; CTS, carpal tunnel syndrome; M20, $20 \mathrm{mg}$ methylprednisolone; M40, $40 \mathrm{mg}$ methylprednisolone; T20, $20 \mathrm{mg}$ triamcinolone; T40, $40 \mathrm{mg}$ triamcinolone. 
Table 3 Comparison of pre- and posttreatment electrodiagnostic values in each group and between groups

\begin{tabular}{|c|c|c|c|}
\hline & Pre & Post & $\begin{array}{l}P \text {-value } \\
\text { (paired } t \text {-test) }\end{array}$ \\
\hline \multicolumn{4}{|c|}{ SNAP latency (ms) } \\
\hline T20 $(n=18)$ & $4.4 \pm 0.6$ & $4.2 \pm 0.4$ & $0.001 *$ \\
\hline T40 $(n=20)$ & $4.4 \pm 0.8$ & $4 \pm 0.6$ & $0.001 *$ \\
\hline$M 20(n=17)$ & $4.2 \pm 0.4$ & $4 \pm 0.3$ & $0.004 *$ \\
\hline$M 40(n=18)$ & $4.7 \pm 0.9$ & $4.4 \pm 0.7$ & $0.003^{*}$ \\
\hline$P$ (ANOVA) & 0.185 & 0.108 & \\
\hline \multicolumn{4}{|c|}{ CMAP latency (ms) } \\
\hline$T 20(n=18)$ & $4.3 \pm 0.6$ & $4.2 \pm 0.7$ & $0.00 I^{*}$ \\
\hline T40 $(n=20)$ & $4.5 \pm 0.8$ & $4 \pm 0.6$ & $0.001 *$ \\
\hline$M 20(n=17)$ & $4.5 \pm 0.8$ & $4 \pm 0.5$ & $0.006^{*}$ \\
\hline$M 40(n=18)$ & $4.7 \pm 0.9$ & $4.4 \pm 0.8$ & $0.00 I^{*}$ \\
\hline$P$ (ANOVA) & 0.685 & 0.366 & \\
\hline \multicolumn{4}{|c|}{ CMAP amplitude (mV) } \\
\hline$T 20(n=18)$ & $5.8 \pm 1$ & $6.3 \pm 1.4$ & 0.117 \\
\hline T40 $(n=20)$ & $6 \pm 1$ & $6.6 \pm 1.3$ & $0.032 *$ \\
\hline$M 20(n=17)$ & $6.5 \pm 1.3$ & $7 \pm 1.8$ & 0.289 \\
\hline$M 40(n=18)$ & $5.9 \pm 1$ & $6.2 \pm 0.8$ & 0.127 \\
\hline$P($ ANOVA $)$ & 0.215 & 0.316 & \\
\hline \multicolumn{4}{|c|}{ Motor NCV $(\mathrm{m} / \mathrm{s})$} \\
\hline$T 20(n=18)$ & $53.7 \pm 5.7$ & $56.3 \pm 7.1$ & $0.025^{*}$ \\
\hline T40 $(n=20)$ & $51.6 \pm 5.6$ & $54.4 \pm 5.9$ & $0.00 I^{*}$ \\
\hline$M 20(n=17)$ & $54.3 \pm 4.3$ & $56.5 \pm 4.3$ & $0.016 *$ \\
\hline$M 40(n=18)$ & $51.3 \pm 4.5$ & $54.0 \pm 5.6$ & $0.00 I^{*}$ \\
\hline$P$ (ANOVA) & 0.203 & 0.484 & \\
\hline
\end{tabular}

Note: *indicates significant $P$-value.

Abbreviations: CMAP, compound motor action potential; M20, $20 \mathrm{mg}$ methylprednisolone; M40, $40 \mathrm{mg}$ methylprednisolone; NCV, nerve conduction velocity; T20, $20 \mathrm{mg}$ triamcinolone; T40, $40 \mathrm{mg}$ triamcinolone; SNAP, sensory nerve action potential.

In Table 3, all electrodiagnostic parameters including SNAP latency, CMAP amp or latency, and NCV are compared in each group (pre- and posttreatment values) and among the four groups, using paired $t$-test and one-way ANOVA, respectively.

SNAP "latency" improved in all the groups after the treatment; there was no significant difference among them in their improvement. Similarly, CMAP latency and subsequently NCV values decreased significantly in all the groups at a follow-up period of 3 months, and no significant difference among them was found. On the other hand, CMAP amp improved significantly only in T40 group $(P=0.032)$, while there was no significant improvement in the other groups.

Among other nonelectrodiagnostic variables (Table 2), PFGS improved significantly in all the groups, except for M20. Similar improvements were found regarding the pain intensity (VAS) in all the four groups. Furthermore, throughout combining the relative groups, we reclassified
Table 4 Comparison of posttreatment values of clinical variables between cumulative groups

\begin{tabular}{|c|c|c|}
\hline & Post & $P$-value (Student's t-test) \\
\hline \multicolumn{3}{|l|}{ PFGS (kg) } \\
\hline$T(n=38)$ & $31.9 \pm 12.1$ & \multirow[b]{2}{*}{0.80} \\
\hline$M(n=35)$ & $32.4 \pm 15.3$ & \\
\hline $20 \mathrm{mg}(\mathrm{n}=35)$ & $33.07 \pm 15.7$ & \multirow[b]{2}{*}{0.63} \\
\hline $40 \mathrm{mg}(\mathrm{n}=38)$ & $31.34 \pm 13.2$ & \\
\hline \multicolumn{3}{|c|}{ VAS (0-I 0 scale) } \\
\hline$T(n=38)$ & $3.84 \pm 2.1$ & \multirow[b]{2}{*}{0.53} \\
\hline$M(n=35)$ & $3.53 \pm 2.1$ & \\
\hline $20 \mathrm{mg}(\mathrm{n}=35)$ & $4.05 \pm 1.8$ & \multirow[b]{2}{*}{0.14} \\
\hline $40 \mathrm{mg}(\mathrm{n}=38)$ & $3.36 \pm 2.2$ & \\
\hline \multicolumn{3}{|c|}{ SSS ( I I-55 scale) } \\
\hline$T(n=38)$ & $23.5 \pm 10.5$ & \multirow[b]{2}{*}{0.67} \\
\hline$M(n=35)$ & $22.6 \pm 7.7$ & \\
\hline $20 \mathrm{mg}(\mathrm{n}=35)$ & $23.88 \pm 9.3$ & \multirow[b]{2}{*}{0.50} \\
\hline $40 \mathrm{mg}(\mathrm{n}=38)$ & $22.44 \pm 9.1$ & \\
\hline \multicolumn{3}{|c|}{ FSS (8-40 scale) } \\
\hline$T(n=38)$ & $14.0 \pm 4.9$ & \multirow[b]{2}{*}{0.27} \\
\hline$M(n=35)$ & $15.3 \pm 5.3$ & \\
\hline $20 \mathrm{mg}(\mathrm{n}=35)$ & $16.46 \pm 5.7$ & \multirow[b]{2}{*}{$0.005^{*}$} \\
\hline $40 \mathrm{mg}(\mathrm{n}=38)$ & $13.05 \pm 4.4$ & \\
\hline
\end{tabular}

Note: *indicates significant $P$-value.

Abbreviations: FSS, functional status scale; M, methylprednisolone; PFGS, painfree grip strength; SSS, symptom severity scale; T, triamcinolone.

data into two larger groups of $20 \mathrm{mg}$ vs $40 \mathrm{mg}$ injections to achieve a more valid analysis. Among all the variables, only FSS showed a remarkable superiority in favor of 40-mg group. In a similar way, we compared two cumulative groups of triamcinolone vs methylprednisolone, with 38 and 35 members, respectively. However, none of the two substances demonstrated any significant superiority (Table 4). Eventually it is noteworthy that this study had neither minor nor major complications related to local steroid injection.

\section{Discussion}

CSI, an established therapeutic method, due to the rapid effects and safety, is usually considered before surgery, for patients with nonsevere CTS, particularly in whom the other conservative therapies did not relieve the symptoms. ${ }^{8,24,25}$ The better outcomes with local steroid injection in comparison with placebo, oral medications or splinting have been proven in several studies spanning over the three decades. ${ }^{26,27}$ However, the type of corticosteroid and its appropriate dosage remain controversial.

Some authors have compared the efficacy and safety of popular corticosteroids at different dosages in CTS patients. ${ }^{13-15} \mathrm{O}$ 'Gradaigh and Merry compared the outcomes 
of local injection of long- and short-acting corticosteroids at different dosages. After 6 weeks and also 6 months, the efficacy of administrating $25 \mathrm{mg}$ hydrocortisone was comparable to that of $100 \mathrm{mg}$ hydrocortisone or $20 \mathrm{mg}$ triamcinolone. Therefore, the authors suggested lower dosage of corticosteroid, regarding potentially lower toxicity. However, their study had some shortcomings such as using overall lower doses in comparison with other trials, lack of power to detect significant differences, and employing a very complex design. ${ }^{15}$

Recently, Salman Roghani et al compared the effectiveness of local injections of 40 and $80 \mathrm{mg}$ triamcinolone in a $3 \mathrm{~mL}$ solution of lidocaine and normal saline (NS) among CTS patients in Iran. In the control group, only $1 \mathrm{~mL}$ of $2 \%$ lidocaine and $2 \mathrm{~mL}$ of NS were administered. The authors found no remarkably significant difference between the two groups; then, they concluded that hydrodissection is more important than anti-inflammatory effects of corticosteroids. ${ }^{13}$ However, because of lidocaine injection in all the groups and other nonspecific benefits of participants for being in an RCT, this effect might not be so "pure", as the authors have said.

In another trial in the Netherlands, Dammers et al locally injected 20, 40, and $60 \mathrm{mg}$ hydrocortisone for patients with CTS. Eventually no significant difference was found between the groups after 12-month follow-up; however, after 6 months, the symptoms were relieved in $56 \%, 53 \%$, and $73 \%$ of patients of 20-, 40-, and 60-mg groups, respectively. ${ }^{14}$ In this study, it was demonstrated that local CSI led to pain relief, improved SSS and FSS scores, increased grip, and improved electrophysiologic parameters (SNAP, CMAP, and $\mathrm{NCV}$ ). Because of some limitations in our trial such as limited sample size and short-term follow-up, only small differences between the investigated groups were found, which should be cautiously considered. Local injection of $20 \mathrm{mg}$ methylprednisolone did not affect PFGS, while PFGS significantly increased in the other three groups. In addition, there were some findings in favor of better outcomes in groups with an injection of $40 \mathrm{mg}$ corticosteroids; 1) FSS was significantly better in 40-mg group compared with $20 \mathrm{mg}$ injection of both substances, especially triamcinolone, after 3-month followup $(P=0.005)$, and 2) CMAP amp improved significantly only in T40 group $(P=0.032)$, while there was no significant improvement in the other groups. It is noteworthy that, in the present study, there were no complications related to local steroid injection and lack of a control group.

\section{Conclusion}

Based on the findings of the current study, local injection of long-acting and short-acting corticosteroids at different dosages can result in a substantial improvement in functional status and symptom relief in patients with CTS in short-term follow-up. However, higher dosage of both substances, especially triamcinolone, was associated with better outcomes in functional status.

\section{Data sharing statement}

The authors do not intend to share substantial data of this study, but they are ready to share de-identified file of substantial data in excel format and all other study-related documents, at any specific time for any period, if the editorial board requires.

\section{Acknowledgments}

This RCT had no funding source. This article has been extracted from the thesis written by Dr Shahab Bagheri, School of Medicine, Shahid Beheshti University of Medical Sciences (Registration No. 65). The authors would also like to express their deep sense of gratitude and respect to the academic supervisor, former chief, and chairman of Akhtar Educational Hospital, Professor Shahriar Bagheri for infusing confidence and a sense of excitement and inspiring us in our work through his encouragement and guidance.

\section{Disclosure}

The authors report no conflicts of interest in this work.

\section{References}

1. Kim PT, Lee HJ, Kim TG, Jeon IH. Current approaches for carpal tunnel syndrome. Clin Orthop Surg. 2014;6(3):253-257.

2. Dale AM, Harris-Adamson C, Rempel D, et al. Prevalence and incidence of carpal tunnel syndrome in US working populations: pooled analysis of six prospective studies. Scand J Work Environ Health. 2013;39(5): 495-505.

3. Luckhaupt SE, Dahlhamer JM, Ward BW, Sweeney MH, Sestito JP, Calvert GM. Prevalence and work-relatedness of carpal tunnel syndrome in the working population, United States, 2010 National Health Interview survey. Am J Ind Med. 2013;56(6):615-624.

4. Fowler JR, Gaughan JP, Ilyas AM. The sensitivity and specificity of ultrasound for the diagnosis of carpal tunnel syndrome: a meta-analysis. Clin Orthop Relat Res. 2011;469(4):1089-1094.

5. Huisstede BM, Hoogvliet P, Randsdorp MS, Glerum S, van Middelkoop M, Koes BW. Carpal tunnel syndrome. Part I: effectiveness of nonsurgical treatments--a systematic review. Arch Phys Med Rehabil. 2010;91(7):981-1004.

6. Turner A, Kimble F, Gulyás K, Ball J. Can the outcome of open carpal tunnel release be predicted? A review of the literature. ANZ J Surg. 2010;80(1-2):50-54.

7. Aroori S, Spence RA. Carpal tunnel syndrome. Ulster Med J. 2008;77(1):6-17.

8. Huisstede BM, Fridén J, Coert JH, Hoogvliet P; European HANDGUIDE Group. Carpal tunnel syndrome: hand Surgeons, hand therapists, and Physical medicine and rehabilitation physicians agree on a multidisciplinary treatment guideline - results from the European HANDGUIDE study. Arch Phys Med Rehabil. 2014;95(12): 2253-2263.

9. Rayegani SM, Raeissadat SA, Heidari S, Moradi-Joo M. Safety and Effectiveness of Low-Level Laser Therapy in Patients With Knee Osteoarthritis: A Systematic Review and Meta-analysis. J Lasers Med Sci. 2017;8(Suppl 1):S12-S19. 
10. Raeissadat SA, Reza-Soltani Z. Study of long term effects of laser therapy versus local corticosteroid injection in patients with carpal tunnel syndrome. J Lasers Med Sci. 2010;1:24-30.

11. Dinarvand V, Abdollahi I, Raeissadat SA, Mohseni Bandpei MA, Babaee M, Talimkhani A. The effect of scaphoid and Hamate mobilization on treatment of patients with carpal tunnel syndrome. Anesth Pain Med. 2017;7(5):e14621.

12. Raeissadat SA, Rayegani SM, Rezaei S, et al. The effect of polarized polychromatic noncoherent light (bioptron) therapy on patients with carpal tunnel syndrome. J Lasers Med Sci. 2014;5(1):39-46.

13. Salman Roghani R, Holisaz MT, Tarkashvand M, et al. Different doses of steroid injection in elderly patients with carpal tunnel syndrome: a tripleblind, randomized, controlled trial. Clin Interv Aging. 2018;13:117-124.

14. Dammers JW, Roos Y, Veering MM, Vermeulen M. Injection with methylprednisolone in patients with the carpal tunnel syndrome: a randomised double blind trial testing three different doses. $J$ Neurol. 2006;253(5):574-577.

15. O'Gradaigh D, Merry P. Corticosteroid injection for the treatment of carpal tunnel syndrome. Ann Rheum Dis. 2000;59(11):918-919.

16. Raeissadat SA, Shahraeeni S, Sedighipour L, Vahdatpour B. Randomized controlled trial of local progesterone vs corticosteroid injection for carpal tunnel syndrome. Acta Neurol Scand. 2017;136(4):365-371.

17. Bahrami MH, Shahraeeni S, Raeissadat SA. Comparison between the effects of progesterone versus corticosteroid local injections in mild and moderate carpal tunnel syndrome: a randomized clinical trial. $B M C$ Musculoskelet Disord. 2015;16(1):322.
18. Raeissadat SA, Karimzadeh A, Hashemi M, Bagherzadeh L. Safety and efficacy of platelet-rich plasma in treatment of carpal tunnel syndrome; a randomized controlled trial. BMC Musculoskelet Disord. 2018;19(1):49.

19. Marshall S, Tardif G, Ashworth N. Local corticosteroid injection for carpal tunnel syndrome. Cochrane Database Syst Rev. 2007;2:CD001554.

20. Waterbrook AL, Balcik BJ, Goshinska AJ. Blood glucose levels after local musculoskeletal steroid injections in patients with diabetes mellitus: a clinical review. Sports Health. 2017;9(4):372-374.

21. Hagebeuk EE, de Weerd AW. Clinical and electrophysiological followup after local steroid injection in the carpal tunnel syndrome. Clin Neurophysiol. 2004;115(6):1464-1468.

22. Wolny $T$, Linek P. Is manual therapy based on neurodynamic techniques effective in the treatment of carpal tunnel syndrome? A randomized controlled trial. Clin Rehabil. Epub 2018 Oct 11.

23. Bland JD. A neurophysiological grading scale for carpal tunnel syndrome. Muscle Nerve. 2000;23(8):1280-1283.

24. Boyer MI. Corticosteroid injection for carpal tunnel syndrome. J Hand Surg Am. 2008;33(8):1414-1416.

25. Stark H, Amirfeyz R. Cochrane corner: local corticosteroid injection for carpal tunnel syndrome. J Hand Surg Eur Vol. 2013;38(8):911-914.

26. Çeliker R, Arslan S, Inanici F. Corticosteroid injection vs. nonsteroidal antiinflammatory drug and splinting in carpal tunnel syndrome. Am J Phys Med Rehabil. 2002;81(3):182-186.

27. Herskovitz S, Berger AR, Lipton RB. Low-dose, short-term oral prednisone in the treatment of carpal tunnel syndrome. Neurology. 1995;45(10):1923-1925.
Journal of Pain Research

\section{Publish your work in this journal}

The Journal of Pain Research is an international, peer reviewed, open access, online journal that welcomes laboratory and clinical findings in the fields of pain research and the prevention and management of pain. Original research, reviews, symposium reports, hypothesis formation and commentaries are all considered for publication.

\section{Dovepress}

The manuscript management system is completely online and includes a very quick and fair peer-review system, which is all easy to use. Visit http://www.dovepress.com/testimonials.php to read real quotes from published authors. 